\title{
artigo
}

Sousa, D.M.S.; Neves da Silva, M.R.; Souza, N.F.; Rodrigues da Silva, A.; Barbosa, A.S.; Studart, R.M.B.;

Ventiladores mecânicos usados em pacientes com COVID-19: desafios na desmontagem segura

\section{Ventiladores mecânicos usados em pacientes com COVID-19: desafios na desmontagem segura}

\author{
Mechanical fans used in patients with COVID-19: challenges in safe disassembly \\ Ventiladores mecánicos utilizados en pacientes con COVID-19: retos del desmontaje seguro
}

\begin{abstract}
RESUMO
Objetivo: Descrever os sentimentos dos enfermeiros frente à desmontagem segura dos ventiladores mecânicos usados em pacientes com Covid-19 dentro da unidade de terapia intensiva. Método: Trata-se de um estudo descritivo e exploratório, com abordagem qualitativa. Os dados foram coletados entre março e junho de 2020, por meio de entrevista individual semiestruturada, com uma amostra composta por 13 enfermeiros. Os dados foram submetidos à análise lexical no Software IRAMUTEQ e analisados por meio da Classificação Hierárquica Descendente (CHD) e Análise de Similitude. Resultados: Os discursos apresentaram a insegurança e a discordância dos enfermeiros que atuam na UTI destinada à pacientes com COVID-19, expondo os profissionais ao risco de contaminação. Conclusão: Mediante a descrição de sentimentos dos enfermeiros entrevistados, foi unânime a concordância de que a descentralização no processamento desses artigos contaminados aumenta o risco de disseminação da doença e deixa os trabalhadores mais susceptíveis ao risco de adoecimento.
\end{abstract}

DESCRITORES: Enfermagem; Coronavirus; Riscos ocupacionais.

\section{ABSTRACT}

Objective: To describe the nurses' feelings regarding the safe disassembly of mechanical ventilators used in patients with Covid-19 inside the intensive care unit. Method: This is a descriptive and exploratory study, with a qualitative approach. Data were collected between March and June 2020, through semi-structured individual interviews, with a sample of 13 nurses. The data were submitted to lexical analysis in the IRAMUTEQ Software and analyzed using the Descending Hierarchical Classification (CHD) and Similitude Analysis. Results: The speeches showed the insecurity and disagreement of nurses working in the ICU for patients with COVID-19, exposing professionals to the risk of contamination. Conclusion: Upon describing the feelings of the interviewed nurses, there was unanimous agreement that decentralization in the processing of these contaminated articles increases the risk of spreading the disease and leaves workers more susceptible to the risk of illness.

DESCRIPTORS: Nursing; Coronavirus; Occupational risks.

\section{RESUMEN}

Objetivo: Describir el sentimiento de las enfermeras sobre el desmontaje seguro de los ventiladores mecánicos utilizados en pacientes con Covid-19 dentro de la unidad de cuidados intensivos. Método: Se trata de un estudio descriptivo y exploratorio, con enfoque cualitativo. Los datos se recolectaron entre marzo y junio de 2020, mediante entrevistas individuales semiestructuradas, con una muestra de 13 enfermeras. Los datos se sometieron a análisis léxico en el software IRAMUTEQ y se analizaron utilizando la Clasificación Jerárquica Descendente (CHD) y el Análisis de Similitud. Resultados: Los discursos mostraron la inseguridad y el desacuerdo de los enfermeros que trabajan en la UCI para pacientes con COVID-19, exponiendo a los profesionales al riesgo de contaminación. Conclusión: Al describir los sentimientos de los enfermeros entrevistados, hubo acuerdo unánime en que la descentralización en el procesamiento de estos artículos contaminados aumenta el riesgo de propagación de la enfermedad y deja a los trabajadores más susceptibles al riesgo de enfermedad.

DESCRIPTORES: Enfermeria; Coronavirus; Riesgos laborales.

RECEBIDO EM: 26/01/2021 APROVADO EM: 08/02/2021

\section{Desirée Maria da Silva Sousa}

Enfermeira, Pós-graduanda em Enfermagem em terapia intensiva pela Universidade de Fortaleza (UNIFOR), Hospital Geral de Fortaleza - HGF, Fortaleza, Ceará.

ORCID: 0000-0003-2434-8266 
Maria Regilânia Neves da Silva

Enfermeira, Pós-graduanda em Enfermagem em terapia intensiva pela Universidade de Fortaleza (UNIFOR), Fortaleza, Ceará. ORCID: 0000-0002-3967-5596

\section{Nathália França Souza}

Enfermeira, Pós-graduanda em Enfermagem em terapia intensiva pela Universidade de Fortaleza (UNIFOR), Hospital Geral de Fortaleza - HGF, Fortaleza, Ceará.

ORCID: 0000-0002-8432-1543

\section{Alan Rodrigues da Silva}

Farmacêutico, Mestre em Transplante pela Universidade Estadual do Ceará (UECE), Hospital Geral de Fortaleza - HGF, Fortaleza, Ceará.

ORCID: 0000-0002-9633-363X

\section{Aglauvanir Soares Barbosa}

Enfermeira, Mestre em Enfermagem pela Universidade Internacional da Lusofonia Afro-Brasileira (UNILAB), Hospital Geral de Fortaleza - HGF, Fortaleza, Ceará.

ORCID: 0000-0003-4909-563X

\section{Rita Mônica Borges Studart}

Enfermeira, Doutora em Enfermagem pela Universidade Federal do Ceará (UFC), Universidade de Fortaleza - UNIFOR, Fortaleza, Ceará.

ORCID: 0000-0002-5862-5244

\section{INTRODUÇÃO}

A covid-19 tem uma elevada taxa de disseminação e isso é preocupante, tendo em vista os grandes impactos que a doença vem causando em todo o mundo. São bem elucidadas as evidências da potencial contaminação dos profissionais de saúde em pandemias virais, principalmente em procedimentos geradores de aerossóis $(\mathrm{PGAs})^{1}$.

Diante da natureza contagiosa da covid-19, os profissionais que atuam na linha de frente devem considerar todos os aspectos relevantes para uma abordagem de via aérea segura, tanto para evitar possíveis erros, como para diminuir o risco de contaminação desses profissionais. Recomenda-se participar do procedimento um médico, um enfermeiro e/ou um fisioterapeuta, além de um circulante ${ }^{2}$.

O responsável pela intubação deve ser o membro mais experiente no manejo de vias aéreas de pacientes graves, usar preferencialmente ambiente com pressão negativa e filtro $\mathrm{Hepa}^{3}$. $\mathrm{Na}$ ausência dessas condições, deve-se colocar o paciente em um quarto com portas fechadas, janelas abertas e restringir o número de profissionais durante esses procedimentos ${ }^{1}$.
No cenário da assistência, dentre os profissionais de saúde que estão mais vulneráveis a essa infecção, encontram-se enfermeiros, técnicos e auxiliares de enfermagem, por representarem a maioria nos serviços de saúde e por permanecerem 24 horas prestando cuidados junto ao paciente acometido pela doença ${ }^{4}$.

Atuando na linha de frente e prestando assistência aos pacientes intubados, foi percebida uma rotina de abertura do sistema fechado dos circuitos do ventilador mecânico após o uso pelo paciente com covid-19 dentro da própria unidade. As peças eram desmontadas e acondicionadas em sacos pretos para envio a Central de Material e Esterilização (CME). Diante disso, surgiu um questionamento sobre o risco que os profissionais estariam expostos com a manipulação desses circuitos contaminados.

Desse modo objetivou-se descrever os sentimentos dos enfermeiros frente à desmontagem segura dos ventiladores mecânicos usados em pacientes com Covid-19 dentro da unidade de terapia intensiva.

\section{MÉTODO}

Trata-se de um estudo descritivo e exploratório, com abordagem qualitativa, desenvolvido em uma unidade de terapia intensiva de um hospital público de referência em procedimentos de alta complexidade, localizado na capital do estado do Ceará, vinculado ao Sistema Único de Saúde.

Os indivíduos foram convidados a participar da investigação de acordo com os seguintes critérios de inclusão: ser enfermeiro e atuar na unidade de terapia intensiva destinada a pacientes com diagnóstico de Covid-19. Excluíram-se profissionais em licença de saúde ou em gozo de férias. O fechamento amostral deu-se por saturação, ou seja, quando não havia informação nova nos depoimentos, desse modo participaram da investigação 13 enfermeiros.

Os dados foram coletados no período de março a junho de 2020, por meio de entrevista individual semiestruturada, elaborada e validada pelos próprios pesquisadores, composta por três perguntas norteadoras, contemplando assuntos, como: desmonte e a pré-limpeza do ventilador mecânico após o uso no paciente com COVID-19 dentro da própria unidade, organização do fluxo de desmonte e envio ao Centro de Material e Esterilização (CME) e sentimentos vivenciados sobre a 


\section{artigo}

Sousa, D.M.S.; Neves da Silva, M.R.; Souza, N.F.; Rodrigues da Silva, A.; Barbosa, A.S.; Studart, R.M.B.

Ventiladores mecânicos usados em pacientes com COVID-19: desafios na desmontagem segura

segurança de realizar esse desmonte.

As entrevistas foram realizadas em local privativo, sem interrupções. Os depoimentos foram gravados e transcritos de forma exaustiva na tentativa de gerar indicadores qualitativos e quantitativos. Faz-se necessário a caracterização das classes e posteriormente, a análise do conteúdo de todos os discursos 5 . Para garantir o anonimato, identificaram-se os enfermeiros pela letra $\mathrm{E}$, em seguida da numeração arábica na ordem das entrevistas (E1, E2, E3...).

Os dados foram analisados por meio do software IRAMUTEQ (Interface de $\mathrm{R}$ pour les Analyses Multimensionnelles de Textes et de Questionnaires) (SOUZA et al 2018) ${ }^{6}$. Realizaram-se análises lexicográficas clássicas no Iramuteq para com- preender os dados estatísticos e quantificar as evocações e formas. Obteve-se a Classificação Hierárquica Descendente (CHD) para aferir os dados do dendograma em função das classes geradas, considerando as palavras com $\mathrm{X}^{2}>3,84(\mathrm{p}<0,05)$. Posteriormente, executada a Análise de Similitude, que a partir da teoria dos grafos foi capaz de identificar as ocorrências entre palavras e sua conexidade.

$\mathrm{O}$ estudo foi submetido à apreciação do comitê de ética e pesquisa (CEP) do Hospital Geral de Fortaleza, o qual recebeu aprovação com número 4.049.919, sob o CAAE 31452620.5.0000.5040. O estudo respeitou todas as etapas das diretrizes e normas de pesquisa envolvendo seres humanos da Resolução 466/12 do

\section{Figura 1 - Dendrograma da Classificação Hierárquica Descendente.}

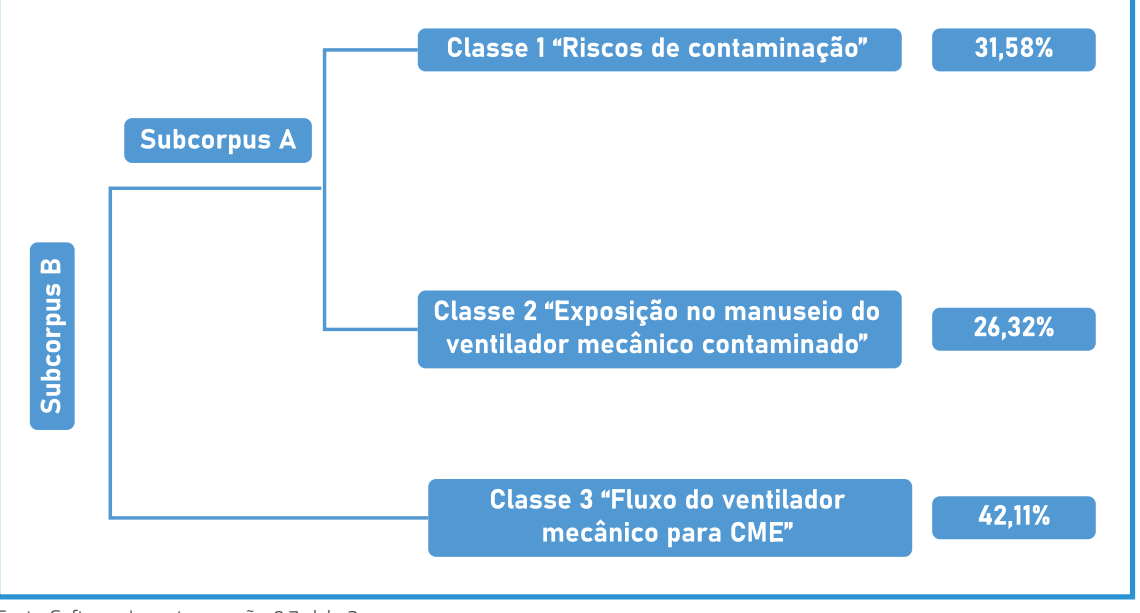

Fonte: Software Iramuteq, versão: 0.7 alpha2

\section{Figura 2 - Organograma de classes a partir das entrevistas com os enfermeiros.}

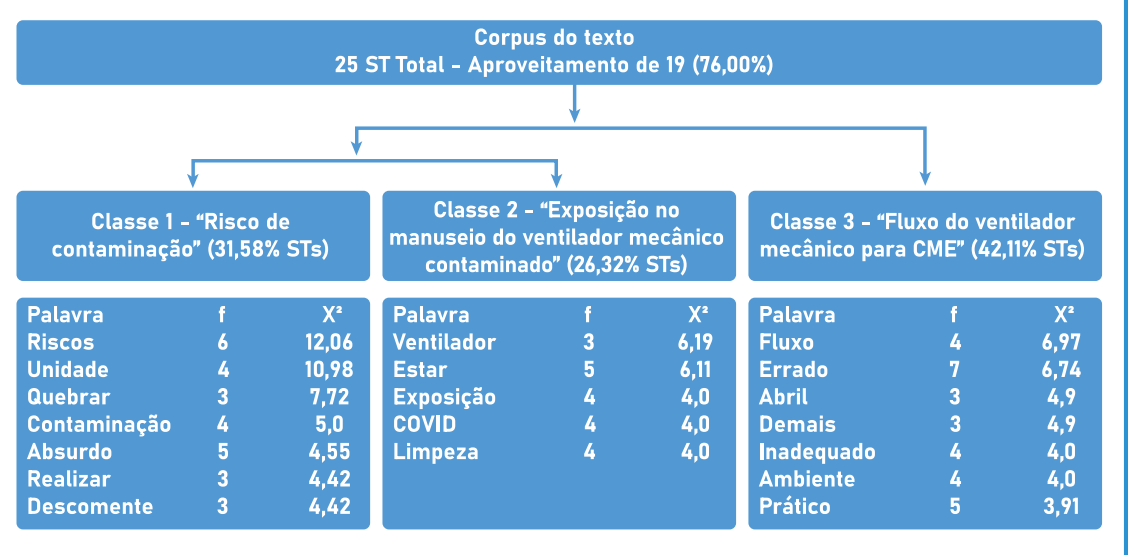

Fonte: Elaboração própria.
Conselho Nacional de Saúde. Foi requerido aos enfermeiros a leitura e assinatura do Termo de Consentimento Livre Esclarecido (TCLE).

\section{RESULTADOS}

O corpus geral foi constituído por 13 textos, separados em 25 segmentos de texto (ST), com aproveitamento de 19 STs $(76,00 \%)$. Emergiram 673 ocorrências (palavras, formas ou vocábulos), sendo 214 palavras distintas e 112 com uma única ocorrência. $\mathrm{O}$ conteúdo analisado foi categorizado em três classes: Classe 1 - "Risco de contaminação", com 06 ST (31,58\%); Classe 2 - "Exposição no manuseio do ventilador mecânico contaminado", com 05 ST (26,32\%) e a Classe 3 - "Fluxo incorreto para CME no transporte do ventilador mecânico", com 08 ST $(42,11 \%)$ (ver figura 1$)$.

Com o intuito de melhor ilustrar as palavras no corpus textual em suas referentes classes, organizou-se um diagrama de classes com exemplos de palavras de cada classe avaliadas por meio do teste qui-quadrado $\left(\mathrm{X}^{2}\right)$. Nele emergem as evocações que apresentam vocabulário semelhante entre si e vocabulário diferente das outras classes. Em seguida serão apresentadas, operacionalizadas e exemplificadas cada uma dessas classes encontradas por meio da análise de Classificação Hierárquica Descendente (ver figura 2).

\section{Classe 1 - "Risco de contaminação"}

Compreende $31,58 \%(f=06 \mathrm{ST})$ do corpus total analisado. Constituída por palavras e radicais no intervalo entre $\mathrm{x}^{2}=$ 4,05 (Não) e x ${ }^{2}=12,06$ (Risco).

Essa classe refere-se à percepção dos enfermeiros entrevistados, após assumir tal atribuição. Relatam ser uma conduta que vai contra as recomendações de segurança preconizada pelo os órgãos competentes de saúde.

Foi observado que muitos profissionais não receberam capacitação técnica para executar o desmonte dos ventiladores, ficando assim mais expostos à contaminação e disseminação da doença. 
[...] não concordo! Absurdo, vai aumentar o risco de contaminação.... nossa segurança... vamos quebrar o lacre das traqueias contaminadas na unidade. (E4)

Esse desmonte é um absurdo, está sendo realizado por profissionais de enfermagem não treinados. Fere a biossegurança aumentando os riscos, não tem segurança [...]. (E7) [...] sem segurança, muitos profissionais não são treinados e acabam fazendo de qualquer maneira se expondo a infecção.. (E10)

$O$ desmonte do ventilador mecânico e pré limpeza, não deve ser realizado na própria unidade, essa manipulação é um absurdo pela exposição e risco para o profissional [...]. (E11)

\section{Classe 2 - "Exposição no manuseio do ventilador mecânico contaminado"}

Compreende 26,32\% $(f=05$ ST) do corpus total analisado. Constituída por palavras e radicais no intervalo entre $\mathrm{x}^{2}=$ 4,0 (Limpeza) e $x^{2}=6,19$ (Ventilador).

Essa classe traz questões relacionadas à percepção dos enfermeiros frente à exposição ao manuseio de ventiladores mecânicos contaminados dentro da própria unidade de terapia intensiva. Relatam preocupação e descontentamento com tal atividade, mostrando-se inseguros.

É unânime a concordância de que essa prática vai contra as recomendações sanitárias em vigor no país. Referem ainda que essa exposição é um risco desnecessário que os profissionais estão expostos, pois de acordo com os entrevistados, todo o processo de limpeza deve ser realizado em ambiente específico, citando como responsável o CME.

Não vejo necessidade de desmontar esse ventilador na UTI e colocar o profissional em maior exposição. Está errado, toda limpeza deve ser feita na CME (E2)

Essa prática só aumenta a exposição do profissional ao vírus. Está errado, estudamos na faculdade, toda limpeza deve ser feita na CME [...]. (E3)

Um absurdo, revoltante o desmonte do ventilador contaminado usado em paciente com Covid dentro de uma UTI. (E13)

\section{Classe 3 - "Fluxo do ventilador mecâ- nico para CME"}

Compreende $42,11 \%(f=08$ ST $)$ do corpus total analisado. Constituída por palavras e radicais no intervalo entre $\mathrm{x}^{2}=$ 3,91 (Prático) e $x^{2}=6,97$ (Fluxo).

Essa classe aborda aspectos relacionados ao fluxo de materiais contaminados realizado de maneira incorreta dentro das UTI's. É notório o sentimento de insegurança e insatisfação nos discursos dos enfermeiros entrevistados. Relatam que tal prática é totalmente inadequada. Os profissionais entrevistados afirmaram que desde a faculdade aprenderam que esse tipo de serviço é de responsabilidade do CME, deve ser realizado em ambiente preparado e equipado para tal ação.

A unidade de terapia intensiva não é um ambiente seguro para o desmonte dos ventiladores mecânicos contaminados, tendo em vista que a infecção por covid-19 é uma doença de fácil disseminação e alta transmissibilidade. Todos reconhecem o risco ao qual estão se expondo, além de defender que todo o processamento do material contaminado deve ser feito de forma centralizado no CME.

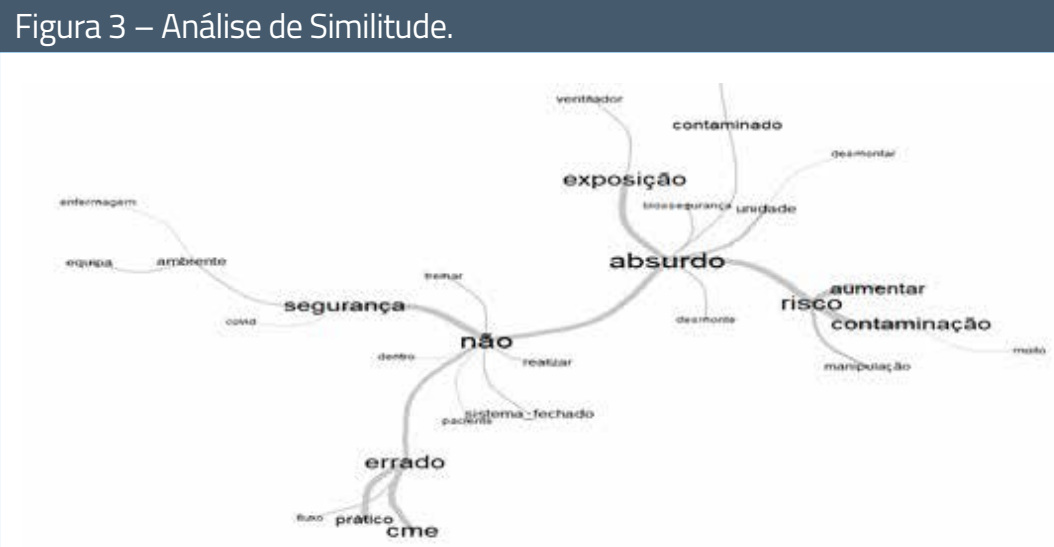

Fonte: Software Iramuteq, versão: 0.7 alpha2
[...] alto grau de transmissibilidade, está errado essa prática, esses fluxos são desrespeitosos e não aceitáveis porque nos expóem ainda mais. (E9)

[...] não tem segurança, o ambiente é inadequado, a equipe não foi treinada, prática errada com fluxo inadequado. (E11)

Um fluxo totalmente errado, manipular dessa forma é errado, pois vamos abrir o sistema fechado muito contaminado no ambiente dos pacientes (E13)

A análise de similitude é fundamentada na teoria dos grafos, na qual é possível identificar as ocorrências entre as palavras e as indicações da conexidade entre as palavras, auxiliando na identificação da estrutura do conteúdo de um corpus textual. Observa-se que a palavra "Não" e "Absurdo" encontram-se no centro dos relatos, e delas ligam-se fortemente com "Segurança", "Exposição" e "Risco" e a partir dessas surgem diversas outras ramificações que fundamenta todo discurso textual (ver figura 3).

\section{DISCUSSÃO}

O Sistema Único de Saúde dispõe de legislações e regimentos de biossegurança que norteiam as práticas assistenciais nos serviços de saúde públicos e privados em todo o país 7 . Por vezes, essas normas e regulamentos não são seguidos confor-

作




\section{artigo}

Sousa, D.M.S.; Neves da Silva, M.R.; Souza, N.F.; Rodrigues da Silva, A.; Barbosa, A.S.; Studart, R.M.B.

me as determinações, e os profissionais se deparam em meio a circunstâncias onde se veem obrigados a não fazer o que é preconizado, colocando em risco sua integridade e de todos que estão envolvidos no processo da assistência ${ }^{8}$.

No cenário atual, os impactos dessa pandemia que o mundo vem enfrentando são imensuráveis, no campo da saúde, as principais deficiências do sistema são a falta de estrutura adequada, escassez de insumos e mão de obra não qualificada, gerando assim uma sobrecarga no sistema, deixando-o prestes a colapsar?.

Os profissionais da saúde que atuam na linha de frente ao combate à covid-19 são mais vulneráveis à infecção ${ }^{10}$. Todo esse contexto em que os trabalhadores estão inseridos e diante das limitações que precisam enfrentar diariamente, resultam em danos físicos e abalos na saúde mental dos mesmos?.

Os serviços assistenciais à saúde devem ser executados sob a premissa da máxima segurança, buscando sempre reduzir ao mínimo a ocorrência de erros ${ }^{11}$. Contudo, no dia-a-dia há eventos e circunstâncias que expõem profissionais à riscos ocupacionais, destacando-se nesse estudo, o processamento de artigos para saúde da assistência ventilatória, onde os profissionais de enfermagem são responsáveis por todo esse processo do desmonte dentro da unidade de terapia intensiva, ficando mais expostos a situações que causam prejuízos à sua saúde ${ }^{12}$.

O ambiente hospitalar é considerado insalubre por conter vários tipos de doenças de natureza contagiosa e por realizar diversos procedimentos que oferecem riscos ocupacionais capazes de causar malefícios à saúde dos trabalhadores ${ }^{12}$. Para que esses riscos sejam minimizados, faz-se necessários que todos os profissionais que atuam na linha de frente no combate a doenças infectocontagiosas recebam treinamentos e capacitações de forma permanente ${ }^{13}$.

O fluxo de material contaminado deve ser unidirecional, com barreiras físicas e/ ou técnicas entre as áreas. $\mathrm{O}$ profissional responsável deve ter registro junto ao seu conselho de classe comprovando a sua ca-

pacidade técnica para desempenhar a função designada e deve também está inserido em programa de educação permanente ${ }^{7}$. O CME tem papel fundamental no processamento dos artigos para a assistência à

Os profissionais da

saúde que atuam na

linha de frente ao

combate à covid-19

são mais vulneráveis

à infecção. Todo esse

contexto em que os

trabalhadores estão

inseridos e diante

das limitaçôes que

precisam enfrentar

diariamente,

resultam em danos

físicos e abalos na

saúde mental dos

mesmos. saúde que atualmente trabalham de forma centralizada seguindo as recomendações de segurança estabelecidas pelas autoridades sanitárias ${ }^{14}$.

Por meio dos depoimentos coletados, evidencia-se que não é fácil para os profissionais que atuam na linha de frente na assistência a pacientes infectados por covid-19, executarem novas rotinas de trabalho sem as condições adequadas. Podemos constatar o quanto estão sendo expostos à doença, colocando em risco suas vidas $\mathrm{e}$ aumentando ainda mais o risco de disseminação da doença $a^{15}$.

Os órgãos sanitários de saúde criam protocolos e recomendam que cada instituição prestadora do cuidado, implementem durante a assistência. No caso da assistência relacionada a ventilação mecânica, é requerido adotar uma sequência de etapas no momento de realizar o processamento dos artigos, compreendendo desde a remoção do equipamento do paciente e encaminhamento para processamento até a sua reutilização. Diante dos depoimentos coletados neste estudo, percebe-se que a descentralização desse serviço pode gerar perda de aerossóis e manipulação inadequada, aumentando assim o risco para contaminação dos profissionais envolvidos.

Logo, é dever de cada instituição promover uma ambiência adequada e segura durante todo o processo do gerenciamento dos artigos de assistência ventilatória contaminados, para assim garantir segurança para toda a equipe ${ }^{16}$.

É responsabilidade e dever do enfermeiro prestar assistência à pessoa, família e coletividade livre de danos decorrentes de imperícia, negligência ou imprudência e que a enfermagem deve garantir assistência com segurança ${ }^{17}$. Por esse motivo é necessário que essa rotina do desmonte dos respiradores contaminados seja revista, readequada e essa atividade possa ser executada em ambiente seguro.

Nesse contexto, a análise de similitude enfatiza que a presença dos profissionais em instituições hospitalares no contexto da covid-19, em que a principal medida preventiva é o distanciamento, o risco de 
contaminação relacionado a atividade laboral eleva-se consideravelmente, em virtude da magnitude de propagação desse vírus, que confirma incisivamente o contexto do risco que esses trabalhadores estão expostos dentro de um cenário de novas rotinas assistenciais ${ }^{18}$.

\section{CONCLUSÃO}

Mediante a descrição de sentimentos dos enfermeiros entrevistados, percebeu-se a grande insatisfação, medo e insegurança frente ao manuseio, desmonte e transporte de ventiladores mecânicos contaminados dentro da unidade de terapia intensiva após o uso por pacientes com covid-19.

Foi unânime a concordância de que a descentralização no processamento desses artigos contaminados aumenta o risco de disseminação da doença e deixa os trabalhadores mais susceptíveis ao risco de adoecimento.

\section{REFERÊNCIAS}

1. Guimarães HP, Schubert DUC, Rodrigues RR, Timerman S, Freitas APR, Corrêa TD, Cunha KA, Bueno M, Santos TM, Moreira NP, Amoroso D, Santos JROG, Carvalho L, Bacal F QM. Recomendações para Intubação Orotraqueal em pacientes portadores de COVID-19. J Chem Inf Model. 2019;53(9):168999. Disponivel em: http://abramede.com.br/wp-content/uploads/2020/06/RECOMENDACOES-IOT-V05-120520.pdf

2. ANVISA. Nota Técnica No 04/2020 GVIMS / GGTES/ANVISA. Orientações para Serviços de Saúde: Medidas de Prevenção e Controle que Devem Ser Adotadas Durante a Assistência aos Casos Suspeitos ou Confirmados de Infecção pelo novo coronavírus (2019- nCoV). Agência Nac Vigilância Sanitária - Anvisa [Internet]. 2020;5(1):1-92. Available from: http:// portal.anvisa.gov.br/documents/33852/271858/Nota+Técni$\mathrm{ca}+\mathrm{n+04-2020+GVIMS-GGTES-ANVISA/.}$

3. Duff JP, Escobedo MB, Pellegrino JL, Charlton N, Hazinski MF. Diretrizes de 2019 da American Heart Association para Ressuscitação Cardiopulmonar e Atendimento. Am Hear Assoc. 2019;JN0992(1):1-17. Disponivel em: https://eccguidelines. heart.org/wp-content/uploads/2019/11/2019-Focused-Updates_Highlights_PTBR.pdf

4. Souza LPS, Souza AG. Enfermagem brasileira na linha de frente contra o novo Coronavírus: quem cuidará de quem cuida? J nurs Heal. 2020;10(1):1-13. https://periodicos.ufpel.edu.br/ ojs2/index.php/enfermagem/article/view/18444/11240

5. Bardin L. Análise de conteúdo. 70th ed. 1979. 1-229 p. Available from: www.edicoes70.pt

6. Souza MAR, Wall ML, Thuler ACMC, Lowen IMV, Peres AM. O uso do software IRAMUTEQ na análise de dados em pesquisas qualitativas. Rev Esc Enferm USP. 2018;52:e03353. https://doi. org/10.1590/s1980-220x2017015003353.

7. BRASIL. Ministério da Saúde. Agência Nacional de Vigilância Sanitária (ANVISA). RESOLUÇÃO No 15, DE 15 DE MARÇO DE 2012. Dispõe sobre requisitos de boas práticas para o processamento de produtos para saúde e dá utras providências. Diário Oficial da União, Brasília, 19 de mar. de 2012ª . Seção 1, p. 43-46.

8. Borgheti SP, Viegas K, Caregnato RCA. Biossegurança no centro de materiais e esterilização: dúvidas dos profissionais*. Rev SOBECC. 2016;21(1):3. https://doi:10.5327/Z14144425201600010002.

9. Barbosa AS, Nascimento C V, Dias LBS, Tiago B, Santo E, Renata CS, et al. Processo de trabalho e cuidado em saúde mental no Centro de Atenção Psicossocial da UERJ na pandemia de COVID-19 Artigo original. Brazilian J Heal Biomed Sci.
2020;19(1):11-9. Disponivel em: https://www.e-publicacoes. uerj.br/index.php/bjhbs/article/view/53527.

10. Oliveira WK de, Duarte E, França GVA de, Garcia LP. Como o Brasil pode deter a COVID-19. Epidemiol e Serv saude Rev do Sist Unico Saude do Bras. 2020;29(2):e2020044. https:// doi: $10.5123 /$ S1679-49742020000200023.

11. Arons MM, Hatfield KM, Reddy SC, Kimball A, James A, Jacobs JR, et al. Presymptomatic SARS-CoV-2 infections and transmission in a skilled nursing facility. N Engl J Med. 2020;382(22):2081-90. https:// doi: 10.1056/NEJMoa2008457

12. Justiniano PM, Eduardo GA, Binotto AHCS, Macedo $\mathrm{Cl}$, Veiga JB, Tognoli T, Mendes SH AA. Riscos ocupacionais e os resíduos de serviços de saúde em centro cirúrgico. Rev SOBECC. 2020;25(1):25-32. https://doi:105327/Z14144425202000010005.

13. Lavich CRP, Terra MG, Mello AL, Raddatz M, Arnemann CT. Ações de educação permanente dos enfermeiros facilitadores de um núcleo de educação em enfermagem. Rev Gauch Enferm. 2017;38(1):e62261. http://dx.doi.org/10.1590/19831447.2017.01.62261.

14. Silva RSDS, Araújo MMZ, Fernandes MA, Batista OMA, Brito BAM, Carvalho NAR. Riscos ocupacionais entre trabalhadores de enfermagem em Unidade de Terapia Intensiva. Rev Bras Med do Trab. 2017;15(3):267-75. https://doi: 10.5327/ Z1679443520170027.

15. LOPES JMM, SOUZA JB. O Papel Das Comissões De Controle De Infecções Hospitalares. Rev Médica Minas Gerais. 2002;(31):105-10. Available from: http://rmmg.org/artigo/detalhes/1581\#

16. Batista MA, Alcântara EC, Paula LKG. Central de ventiladores mecânicos: organização, segurança e qualidade. Rev Bras Ter Intensiva. 2007;19(4):450-5. Available from: https://doi. org/10.1590/S0103-507X2007000400008.

17. Conselho Federal de Enfermagem. Resolução COFEN no 564/2017 - Código de Ética dos Profissionais da Enfermagem [Internet]. Código de Ética dos Profissionais de Enfermagem. 2017. p. 16. Available from: http://www.cofen.gov.br/resolucao-cofen-no-5642017_59145.html.

18. Gallasch CH, Cunha ML da, Pereira LA de S, Silva-Junior JS. Prevenção relacionada à exposição ocupacional do profissional de saúde no cenário de COVID-19. Rev Enferm UERJ [Internet]. 2020;28(0):49596. Available from: https://www.e-publicacoes. uerj.br/index.php/enfermagemuerj/article/view/ 